\author{
EVS25 \\ Shenzhen, China, Nov 5-9, 2010
}

\title{
Electrochemical model and its parameters identification tool for the follow up of batteries ageing
}

\author{
Julien Hafsaoui ${ }^{1}$, Franck Sellier ${ }^{1}$ \\ LMS Imagine, 84 Quai Charles de Gaulle, Lyon, 69006, France \\ E-mail: julien.hafsaoui@lmsintl.com
}

\begin{abstract}
This paper describes research into the definition of experimental protocols, and the development of a numerical model of a battery, associated with a parameterization assistant tool in order to follow battery ageing. This work was carried out as part of the SIMSTOCK program. The experimental characterizations (ageing cycle tests and characterization protocols) are defined in association with the different research lab partners of the project. They were performed on Lithium-ion VL6P cells supplied by Johnson Control SAFT (J.C.S). The characterization protocols are performed at different phases of a program: initially to establish the reference value parameters and periodically, in order to follow all the changes occurring during the ageing of a Li-Ion battery. The different numerical tools presented in this paper are adapted to the LMS-Imagine Lab AMESim simulation platform. The present model makes it possible to simulate the dynamical voltage response of the battery as a function of inlet current and temperature. The identification tool associated is able to determine from a temporal experimental characterization of the battery (U and I), the data necessary for the implementation of the parameters in the model. The assistant tool identifies the parameters of the numerical model at different ages of the Li-Ion battery. Its results are used in order to quantify the influence of ageing phenomena on the performance of the battery considered.
\end{abstract}

Keywords-Battery Electrochemical Model, Parameter Assistant Tool, Ageing

\section{Introduction}

On board Energy Storage Systems (E.S.S) remain one of the major issues to be solved in order to succeed in a massive diffusion of electrified, hybrid, then electric vehicles. Ageing forecasting for E.S.S remains one of the major problems to be solved. The constraints encountered by these vehicles lead to complex powertrain architectures, which require an optimized energy management. A system approach is required, involving simulation of the vehicle and its powertrain components, especially for the E.S.S and among them the batteries and supercapacitors.

This paper first describes the definition of different experimental protocols used to highlight the ageing of batteries and to control the impact of this phenomenon on their performance. Then, the research into the development of a numerical model of a battery, associated with a parameter identification assistant tool is presented. Finally, the first results, obtained by combining these experimental and numerical approaches, concerning the follow up of Lithium-Ion battery ageing are exposed.

This work has been carried out as part of the SIMSTOCK program, which associates research labs, E.S.S manufacturers, component and vehicle manufacturers and software editors with the support of the French Environment and Energy Management Agency (ADEME). The different simulation tools, described in this article, have been developed on the LMS Imagine Lab AMESim simulation platform. 


\section{Ageing tests}

\subsection{General considerations}

This section describes the different experimental protocols used in the SIMSTOCK program. A cycling experimental test is defined in order to accelerate and to highlight the ageing phenomena of a Lithium-Ion battery. A characterization protocol is followed to test, at different phases of the program, the evolution of the behavior of the battery at given ageing conditions. The references [1], [2] and [3] highlight the ageing mechanisms and their precursors involved in the Li-Ion technology. According to these studies, the parameters, which have to be considered as influent in the ageing process and tested in the SIMSTOCK program, are the following ones:

- Temperature of the cell (T)

- Amplitude of State Of Charge (S.O.C) variation $(\Delta$ S.O.C $=$ S.O.C $\max -$ S.O.C min, see Fig.1)

- Amplitude of the discharging pulse current (Id)

- Charge throughput of a microcycle (CT in As)

The different operating conditions ( $\mathrm{T}, \Delta$ S.O.C, Id and $\mathrm{CT}$ ) of the ageing cycle effectuated in the frame of this program are compiled in Table 1. For each ageing parameter, two values (one minimal and one maximal) are tested, which lead to 11 different experiments. It is also noted that an additional cycling test, called validation experiment, is effectuated with the median value for each ageing parameter (see test No.12 in the Table 1).
Table 1: Ageing cycles

\begin{tabular}{ccccc}
\hline & $\mathrm{T}\left({ }^{\circ} \mathrm{C}\right)$ & $\Delta \mathrm{SOC}(\%)$ & $\mathrm{I}(\mathrm{A})$ & $\mathrm{Ct}(\mathrm{As})$ \\
\hline Test 1 & 40 & 20 & 65 & 650 \\
Test 2 & 50 & 40 & 65 & 650 \\
Test 3 & 50 & 20 & 65 & 1300 \\
Test 4 & 40 & 40 & 65 & 1300 \\
Test 5 & 50 & 20 & 130 & 650 \\
Test 6 & 40 & 40 & 130 & 650 \\
Test 7 & 40 & 20 & 130 & 1300 \\
Test 8 & 50 & 40 & 130 & 1300 \\
Test 9 & 50 & 20 & 130 & 1300 \\
Test 10 & 50 & 40 & 130 & 650 \\
Test 11 & 50 & 40 & 65 & 1300 \\
Test 12 & 45 & 30 & 97,5 & 975 \\
\hline
\end{tabular}

\subsection{Cycling experimental tests}

The cycling experimental test, used in the context of the SIMSTOCK program, is represented in Fig.1. It consists of 22 hours of effective cycling and 2 hours of rest in order to preserve an electrode charge homogenization time.

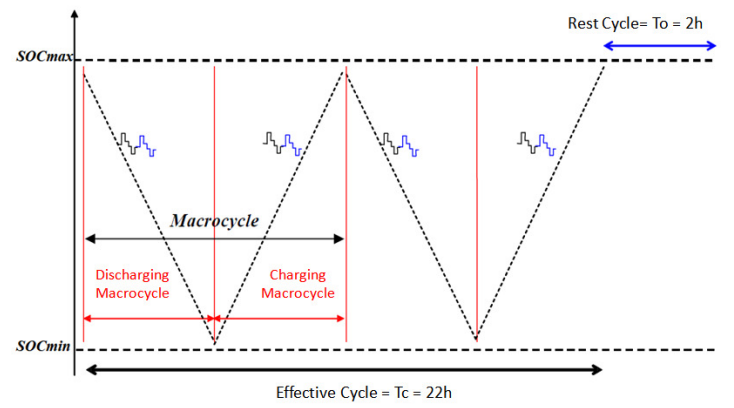

Figure 1: Macrocyle defined in the SIMSTOCK program [4]

The effective cycling is divided into 2 macrocycles, which are defined as a scanning between two values of S.O.C (in Fig.1, it corresponds to the range: [S.O.C min; S.O.C $\max ]$ ). In a macrocycle, there is one phase of discharge and one of charge, which can be seen as a succession of microcycles constituted by charging and discharging pulsations. The different microcycles tested in the context of the SIMSTOCK program are a function of the pair of 
ageing parameters Id and CT chosen. They are represented in Fig.2.
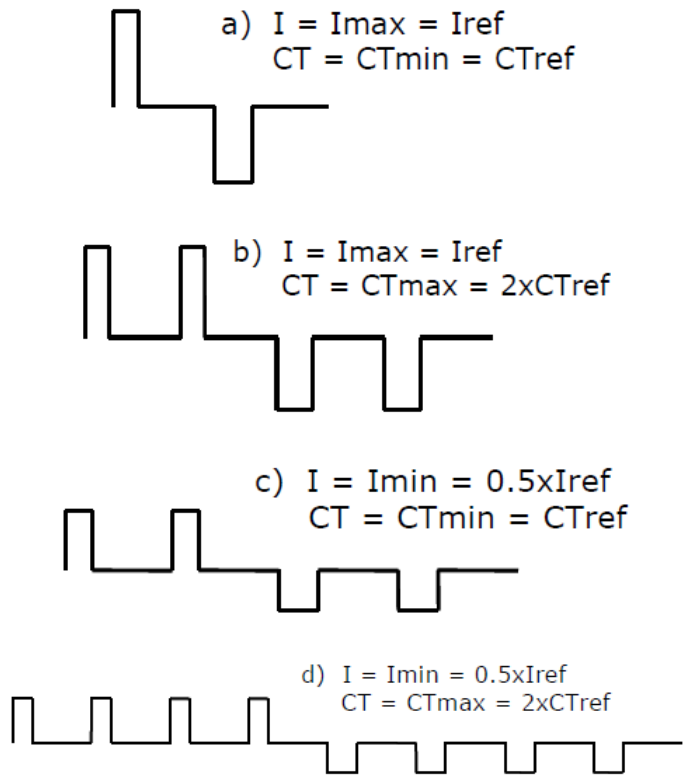

Figure 2: Microcyles defined in the SIMSTOCK program [5]

The cycling experimental tests were performed during ten months with characterization protocols effectuated every six weeks to follow the evolution of the battery behavior.

\subsection{Characterization protocols}

The cells are tested at different phases of the program in order to follow the evolution of the behavior of the battery at given ageing conditions. Initially, the protocol records the reference values of the cells and, periodically, it records the evolution of their performances. The characterization protocol consists in capacity measurement of each cell, an Electrochemical Impedance Spectroscopy (E.I.S) and a time response to a pulse profile, which is represented in Fig.3, at different S.O.C values $(20 \%, 40 \%, 60 \%$ and $80 \%$ ).

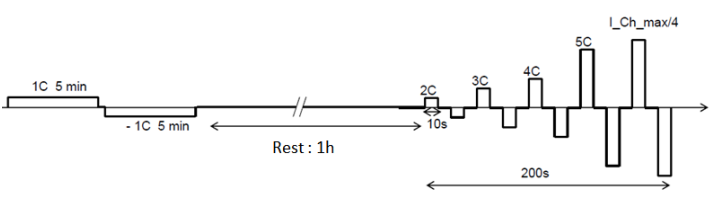

Figure 3: Pulsation characterization protocol defined in the SIMSTOCK program [4]

This characterization protocol contains phases at low current and long time pulse (1C- $5 \mathrm{~min})$ and phases at high current and short time pulse $(5 \mathrm{C}-$ 10s).

\section{Battery simulation}

After presenting the set of experiments defined in the SIMSTOCK program, the simulation tools which have been developed are described in this section.

\subsection{Theory associated to the simulation of battery}

The numerical model has to be able to calculate the dynamical voltage response (U) of the battery as a function of inlet current (I) and temperature (T). The first element to take into account in a battery model is its Open Circuit Voltage (noted O.C.V), which represents the voltage of the battery at its thermodynamical equilibrium. The latter is reached at current equal to zero for a sufficiently long time, which depends on the electrochemical technology under consideration. The second element to simulate in a battery model is the phenomenon of overpotential or overvoltage (noted $\eta_{\text {tot }}$ ). To fully understand this notion, it is necessary to consider a battery which is charged or discharged. This electrochemical system moves away from its equilibrium. If the current is suddenly cut off, a difference between the measured instantaneous voltage and the one obtained after the return to the equilibrium can be observed. This difference of voltage is called overpotential or overvoltage. These last ones are generated by several simultaneous phenomena:

- Charge transfer overvoltage, noted $\eta_{\text {Ст. }}$.

- Diffusion overvoltage, noted $\eta_{\text {diff. }}$

- Ohmic overvoltage, noted $\eta_{\Omega}$.

Consequently, the formula used in the present model to determine the voltage of the battery as a function of the O.C.V and the different overpotentials is as follows:

$$
U=O \cdot C . V-\eta_{t o t}=O \cdot C . V-\eta_{C T}-\eta_{\text {diff }}-\eta_{\Omega}
$$

\subsection{Battery electrochemical model}

The behavioral model, proposed in the present article, uses the approach of equivalent electrical circuits to simulate the different physical phenomena previously mentioned. The circuit is represented in the Fig.4. 


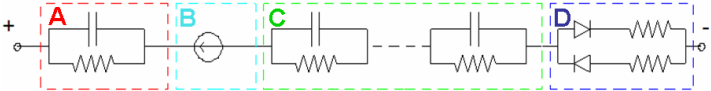

Figure 4: Equivalent electrical circuit.

In the Fig.4, the charge transfer is represented by a parallel RC circuit (frame "A"). The O.C.V is represented by the voltage source (frame " $\mathrm{B}$ "). The ohmic losses are predicted with the two resistances in parallel (frame "D"). The presence of anti-parallel diodes makes it possible to use different values of resistance, according to the direction of the current. Finally, the diffusion is simulated by the serial association of " $n$ " RC parallel circuits (frame " $C$ ").

\subsection{Parameterization assistant tool}

According to the previous presentation of the numerical battery model, different parameters have to be implemented to run a simulation: the values of the O.C.V, the charge and discharge resistances (respectively noted $\mathrm{Rc}$ and $\mathrm{Rd}$ ), the capacitance and the resistance for the charge transfer (respectively noted Rct and Cct). It is noted that the two last parameters can be ignored in the case of temporal simulation. Concerning the " $n$ " $\mathrm{RC}$ circuit modeling the diffusion, they can be considered as not independent each other. Consequently, it is possible to limit the number of parameters to implement the diffusion [6]. Thus, only two parameters have to be introduced and determined to simulate this phenomenon in temporal mode: the Diffusion Time Constant (noted dtc) and the Diffusion Steady State Resistance (noted dssr) [7]. In order to correctly visualize these parameters, a temporal characterization has to be considered as represented in Fig.5.

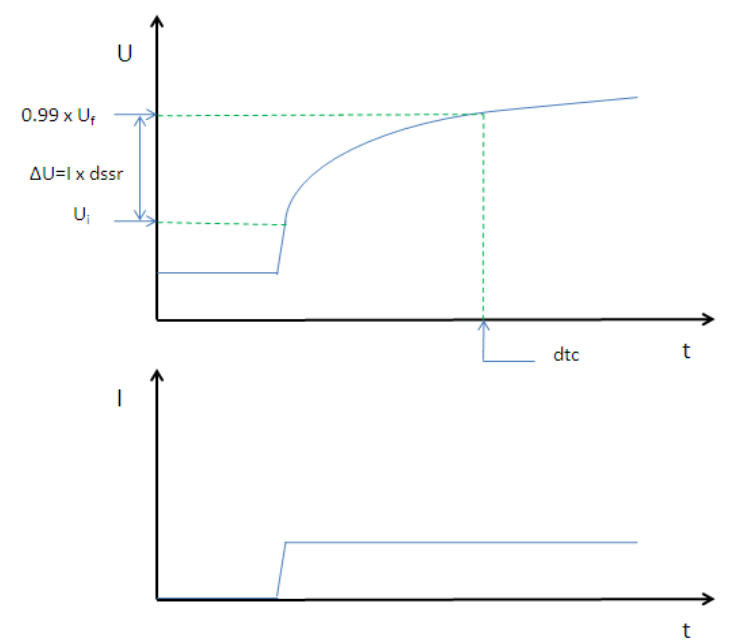

Figure 5: Temporal response of a battery
In the Fig.5, the voltage response to a current pulsation applied to a battery as a function of time is drawn. The voltage before the step added to the ohmic drop due to the current step is noted Ui, whereas the voltage reached at steady-state is noted Uf. The parameter dtc corresponds to the time at which the voltage is equal to $99 \%$ of Uf. The parameter dssr is defined by the following relationship:

$$
d s s r=\frac{U_{f}-U_{i}}{I}=\frac{\Delta U}{I}
$$

The knowledge of different parameters required to implement the present numerical battery model is complex. Moreover, they present dependencies on S.O.C, temperature (T) and possibly current (I). The identification of these dependencies is difficult, because there is no norm or standard procedure to identify these variations. In this context, an identification parameterization assistant tool associated with this numerical model has been developed in order to determine the different parameters and their dependencies. The input data necessary for this assistant are a measurement of an inlet current applied to the battery and its corresponding voltage response. These experimental characterizations have to be effectuated with a large range of S.O.C and current at different temperatures. Then, the assistant tool identifies, "time zone after time zone" (these zones have to be correctly chosen), each parameter (O.C.V, Rc, Rd, dtc and dssr) necessary for the implementation of the battery model in temporal mode. The principles of this parameterization assistant tool are described in [7]. The final aim of this assistant is, after identification, to obtain $2 \mathrm{D}$ and $3 \mathrm{D}$ data files, respectively for the O.C.V and for the other electrical parameters, with their dependences of S.O.C and I at a given temperature. These parameter files are stored in data directly readable by the LMS Imagine Lab AMESim platform.

The process of identification can be divided in three steps: identification of the O.C.V, the ohmic resistances ( $\mathrm{Rc}$ and $\mathrm{Rd}$ ) and, finally, the diffusion parameters (dtc and dssr). The successive results of these steps are represented in Fig.6. To identify the O.C.V, the assistant tool detects automatically the rest zones in the input current data. These zones correspond to the thermodynamical equilibrium of the battery, where the current is close to zero. The assistant determines, graphically the value of the voltage measured for these particular zones. This value identified can 
be considered as corresponding to the O.C.V. The results obtained after this first identification are exposed in the Fig.6a, where the assistant tool can fit (green curve) the experimental curve (black curve) only in the rest zones. The peaks of voltage, caused by current pulsation, are not fitted yet because for these zones, the phenomena of ohmic losses and diffusion are preponderant. Consequently, the second step concerns the identification of ohmic resistances. They are calculated on the temporal zones, where a charge or a discharge sudden variation occurs. The value of $\mathrm{Rc}$ and $\mathrm{Rd}$ is calculated by making the ratio between the difference of the voltage on the current before and after the charge or the discharge pulsation as represented in the equation (3) (see Fig.6b).

$$
R_{\Omega}=\frac{\Delta U}{\Delta I}
$$

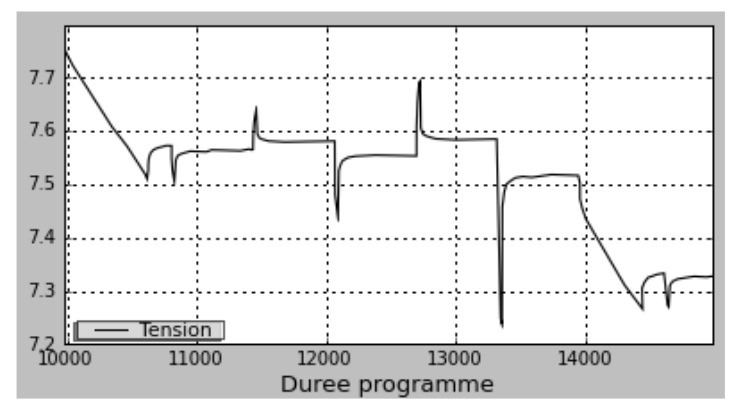

Figure 6: Input data file: voltage response (V) as a function of time (s)

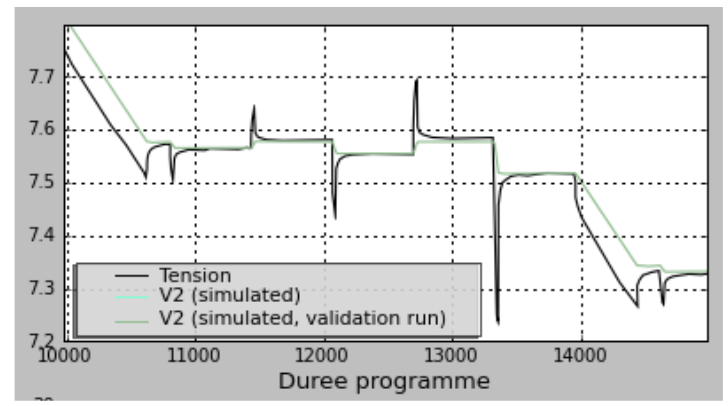

Figure 6a: Comparison between the input data file (black) and the results obtained after the identification of the O.C.V (green)

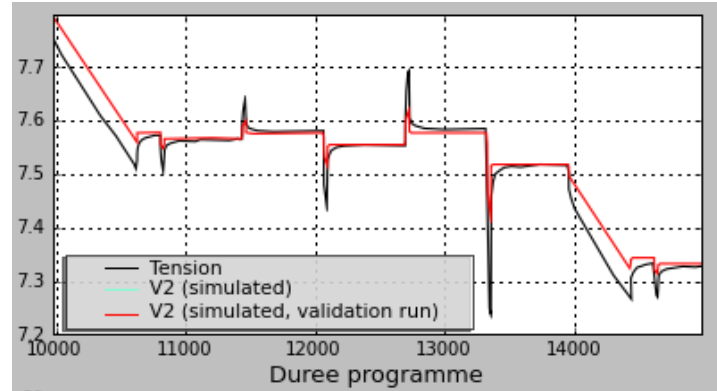

Figure 6b: Comparison between the input data file (black) and the results obtained after the identification of O.C.V and the ohmic resistances (red)

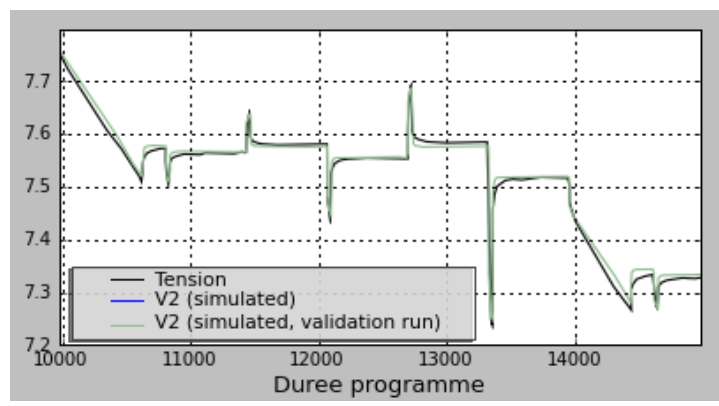

Figure 6c: Comparison between the input data file (black) and the results obtained after the identification of O.C.V, the ohmic resistances and the diffusion parameters (green)

The Fig.6b represents the comparison between the results obtained by the assistant tool (red curve) after this second step in the identification and the experimental curve (black curve). Thus, the peaks of voltage start to be fitted correctly but not totally because, at this stage, the phenomenon of diffusion is not taken into account. The last step of identification consists in determining the dtc and dssr parameters to simulate the diffusion. The method used for this determination is a numerical optimization. At the end of this third identification, the simulated (green) and experimental (black) curves correspond perfectly as represented in Fig.6c.

\subsection{Validation of the parameterization assistant results}

The identification is effectuated on the VL6P LiIon battery supplied by Johnson Control - SAFT (J.C.S). The voltage and current temporal characterizations used for this identification are represented in the Fig.7. 


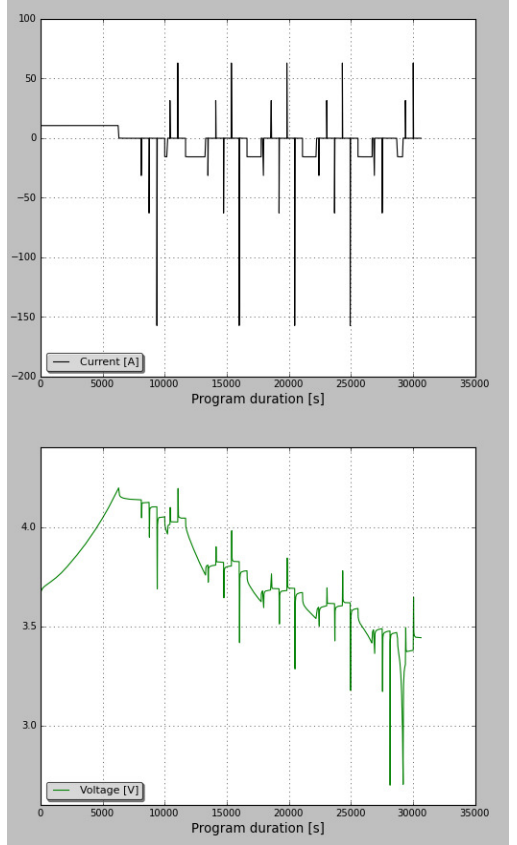

Figure 7: Voltage and current input data used by identification assistant

After this identification, the data files generated by the assistant are introduced in the numerical battery model. To validate the results obtained by the assistant, the inlet current, represented in the Fig.8, is applied to the battery model. The aim of this simulation is to compare the voltage response, obtained by the experiment and by the numerical model, to this inlet current. The comparison is represented in the Fig.9, where the results obtained by experiment and simulation are respectively drawn with the green and red curve. For CPU time reasons, this test is limited to a time range comprised between 3000s and 17000s. The results obtained show that the two curves correspond very well, even in the small time range, where the dynamics are closed. The relative error observed between these two curves is inferior to $2 \%$, which shows the important accuracy of the parameterization assistant tool and the numerical model. The identification done with experimental curves of 30000 s takes 30 minutes, whereas the simulation for a cycle of 14000 s with an interval communication of 1 s (i.e one point is recorded per second) takes 200s, which show the fast performances of the simulation tools described in this present article.

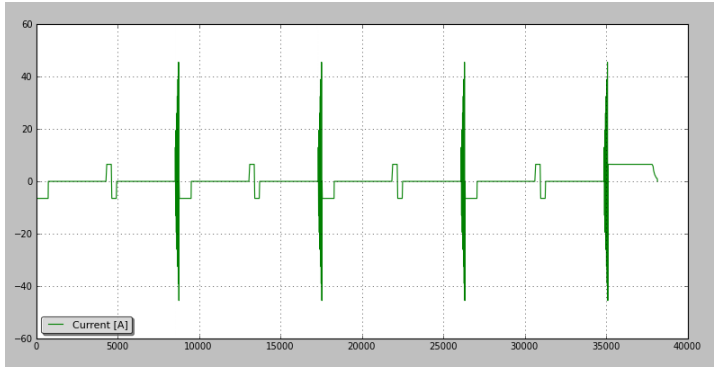

Figure 8: Inlet current input data used in the simulation to validate the identification results
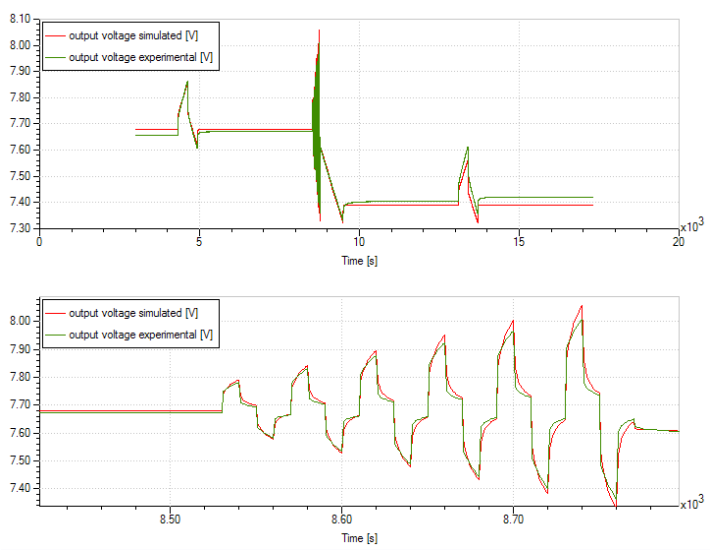

Figure 9: Comparison of voltage response between the experiment (green curve) and the simulation (red curve)

The results presented in this section validate the process used to simulate the behavior of a battery. This process can be divided in two steps. The first consists in determining the necessary parameters for the numerical model. The second one corresponds to the simulation of the battery by using the parameters previously identified.

\section{Battery ageing behavior}

The results presented in this article concern a LiIon battery VL6P with a nominal capacitance of 6,5Ah supplied by Johnson Control - SAFT (J.C.S). The conditions of ageing tests are the following ones: a temperature of $50^{\circ} \mathrm{C}$, a $\Delta$ S.O.C of $40 \%$, an amplitude of the discharging pulse current equal to $130 \mathrm{~A}$ and a charge throughput of $650 \mathrm{As}$, which corresponds to the test No 10 in Table 1. It is noted that with the present ageing conditions, the microcyle used is the one, represented in Fig.2a.

\subsection{Preliminary considerations}

For this set of ageing parameters, the voltage response of the present Li-Ion battery to the 
characterization protocols, recorded at different ages of the battery, are represented in Fig. 10.

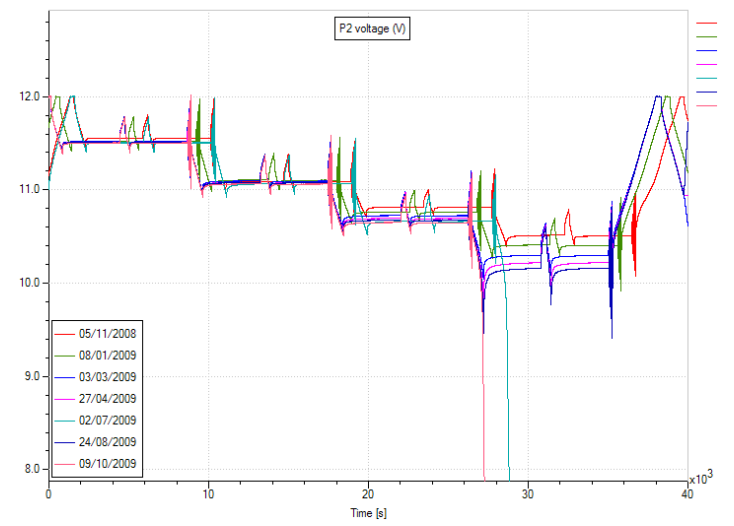

Figure 10: Voltage response of the VL6P battery at different times during their ageing cycle $\left(\mathrm{T}: 50^{\circ} \mathrm{C}\right.$; $\Delta$ S.O.C: $40 \%$; Id: $130 \mathrm{~A}$; CT: 650 AS)

Fig.11 represents the evolution of the measured capacitance as a function of the 7 characterization protocols.

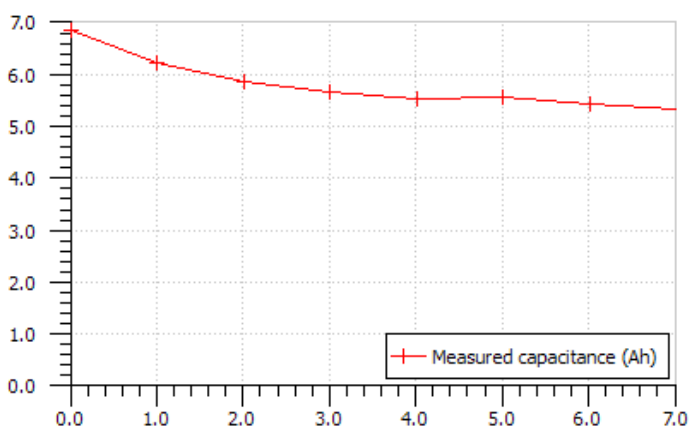

Figure 11: Evolution of the capacitance of the VL6P battery during the ageing cycle (T: $50^{\circ} \mathrm{C} ; \Delta$ S.O.C: 40\%; Id: 130A; CT: 650 AS)

Knowledge of the evolution of the capacitance during the ageing cycle is important. Indeed, this parameter is used during the identification, in particular, to calculate the S.O.C. According to the Fig.11, the capacitance decreases with the ageing cycle, this result confirms that the properties of a Li-Ion battery like its capacitance are influenced by the ageing.

The parameterization assistant makes an identification for each of the previous protocols in order to obtain the evolution of the different parameters (O.C.V, Rc, Rd, dtc and dssr) of the numerical model as a function of S.O.C, current and the age of the Li-Ion VL6P battery. The identification process is limited to a time range between 0 and 25000s because for some protocols, the experimental data are not available for experiment times superior to $27000 \mathrm{~s}$.

\subsection{Identification in function of ageing cycle}

The Fig.12 represents the evolution of the value of O.C.V determined by the assistant tool from the different characterization protocols.

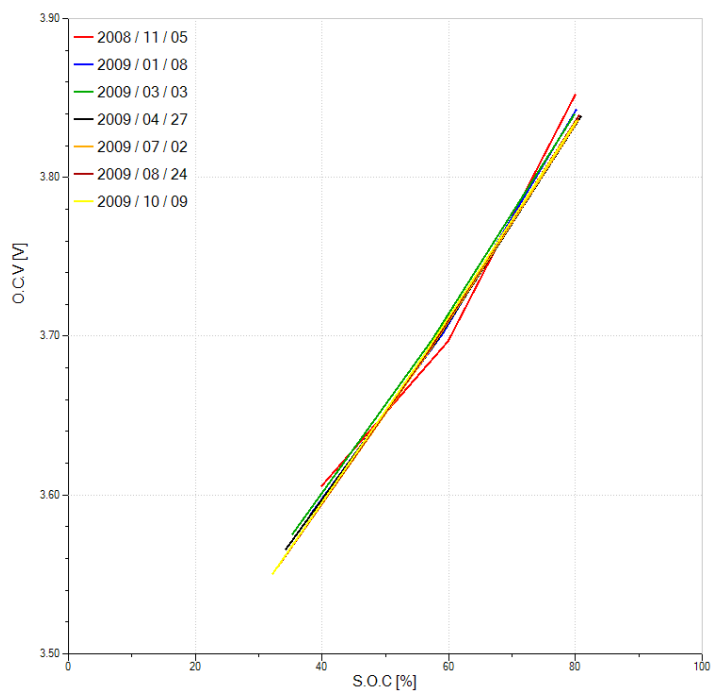

Figure 12: Evolution of the O.C.V of the VL6P battery during the ageing cycle (T: $50^{\circ} \mathrm{C} ; \Delta$ S.O.C: $40 \%$; Id: 130A; CT: $650 \mathrm{AS})$

This Fig. 12 shows that the O.C.V is not affected by the ageing conditions considered, in the range of S.O.C comprised between $20 \%$ and $80 \%$. Nevertheless, it is possible that at low S.O.C, typically below $20 \%$, the ageing parameters have a more marked impact. Indeed, in these conditions of S.O.C, some (not very well understood) phenomena, occur and can be influenced by the ageing of battery. Although, with the electrical approach adopted (see Fig.4) for the present numerical battery model, these phenomena are not taken into account, consequently, for low S.O.C, the model can lose its predictivity because the limits of this physical approach are reached.

Fig.13 represents the values determined by the identification assistant for the ohmic resistances $(\mathrm{Rd}$ and $\mathrm{Rc})$ at the beginning, middle and end of the ageing cycle, respectively displayed in Fig.13a, Fig.13b and Fig.13c.

These maps show that the ohmic resistances are more important for high values of S.O.C and at high positive currents (i.e in charge). These tendencies highlight the energy losses during the charge of the battery because of Joule losses. 
Moreover, for the Li-Ion battery considered, it is noticed that the value of the ohmic resistances increases with the ageing. In the present case, initially, the range of ohmic resistances identified is comprised between $2.25 \mathrm{~m} \Omega$ and $2.55 \mathrm{~m} \Omega$. At the last characterization protocol, the range is between $2.8 \mathrm{~m} \Omega$ and $3 \mathrm{~m} \Omega$.

Fig.14 and Fig.15 represent the values of the diffusion parameters (dssr and dtc) identified with the different characterization protocols. Concerning the dssr, the maps obtained by identification have an identical aspect. The more important values obtained for dssr are located at current close to zero and for low values of S.O.C, in particular when the current is in charge. During the ageing cycle, the range of values of dssr does not change significantly. Concerning the cartographies obtained for the parameter dtc, they present a maximum for a S.O.C comprised between $60 \%$ and $65 \%$ and for a current close to zero. The values of these maxima have no physical sense. They are obtained by extrapolation by the graphical tool used to generate these figures. Indeed, the assistant tool is not able to calculate the diffusion parameters (dssr and dtc) in the zones where the current is close to zero and consequently, the zones where the diffusion is not the preponderant phenomenon. Currently, it is difficult to highlight the changes concerning the diffusion parameters with only one ageing cycle (test No 10 in the Table 1). It would certainly be easier to understand the evolution of these parameters and the physical phenomena involved with the results obtained with the others ageing cycles exposed in the Table 1.

\subsection{Next steps of the SIMSTOCK program}

At this stage we have several maps from each parameter necessary for the implementation of the numerical battery model. These maps give the evolution of these parameters as a function of one ageing cycle (T: $50^{\circ} \mathrm{C}$; S.O.C: $40 \%$; Id: $130 \mathrm{~A}$; CT: $650 \mathrm{~A})$. In the context of SIMSTOCK program, this identification has to be done for all characterization protocols effectuated for each ageing cycle described in Table 1.

It is noted that in the SIMSTOCK program, Ni$\mathrm{MH}$ batteries and supercapacitors supplied by different manufacturers are also studied and submitted to an identical set of experiments and simulation tools.

At the end of all ageing cycles and characterization protocols, a database for all parameters (O.C.V, Rc, Rd, dsssr and dtc) of the numerical model will be available. From this database and thanks to the theory of experiment design, it will be possible to determine the ageing laws for each parameter. The numerical model will be adapted to take into account the evolution of these parameters as a function of the operating conditions of the simulation.

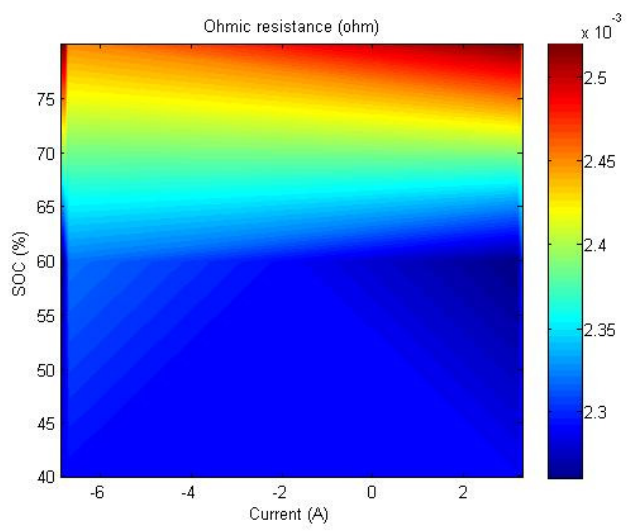

Figure 13a: Ohmic resistances ( $\mathrm{Rd}$ and $\mathrm{Rc}$ ) of the VL6P battery at the beginning of the ageing cycle

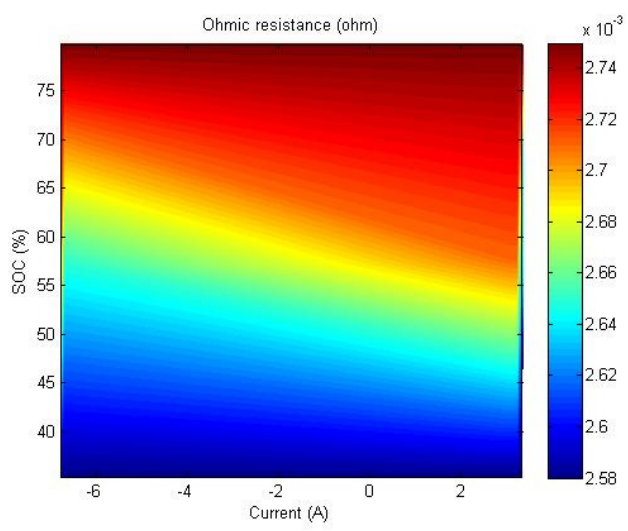

Figure 13b: Ohmic resistances (Rd and Rc) of the VL6P battery at the middle (27 / 04 / 2009) of the ageing cycle

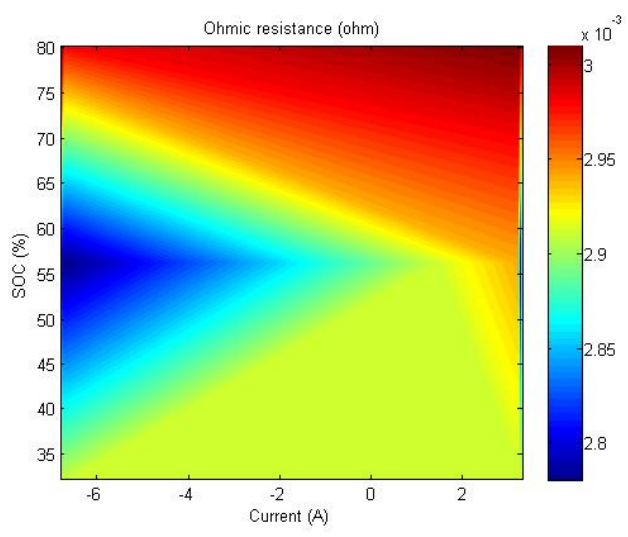

Figure 13c: Ohmic resistances ( $\mathrm{Rd}$ and $\mathrm{Rc})$ of the VL6P battery at the end of the ageing cycle 


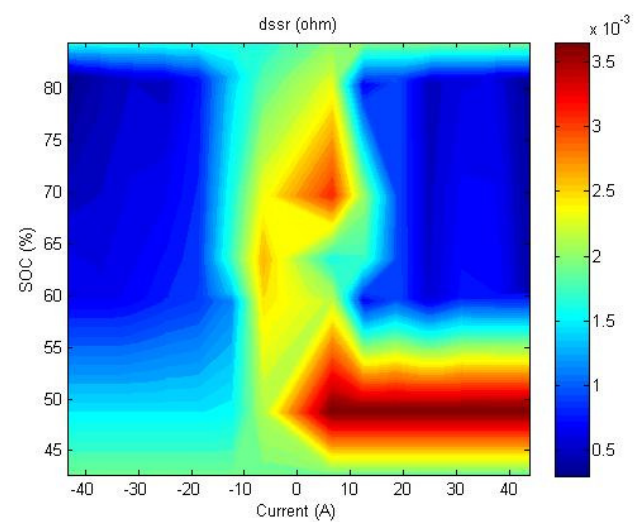

Figure 14a: Diffusion Steady State Resistance (dssr) of the VL6P battery at the beginning of the ageing cycle

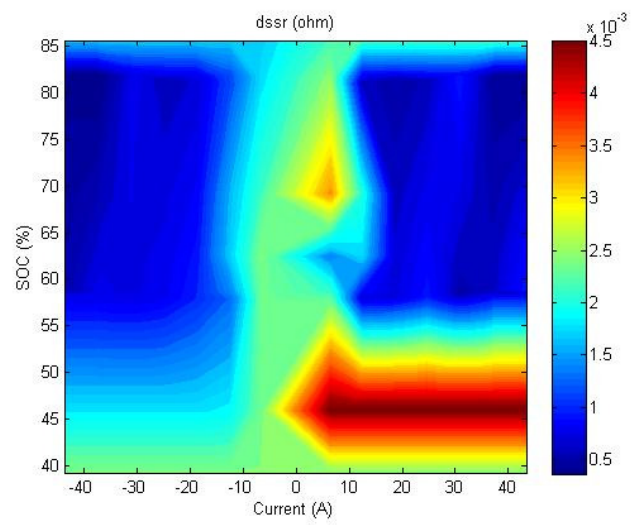

Figure 14b: Diffusion Steady State Resistance (dssr) of the VL6P battery at the middle (27 / 04 / 2009) of the ageing cycle

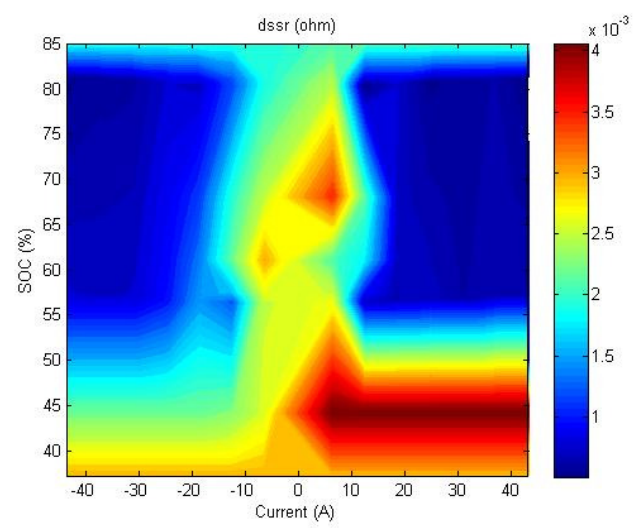

Figure 14c: Diffusion Steady State Resistance (dssr) of the VL6P battery at the end of the ageing cycle

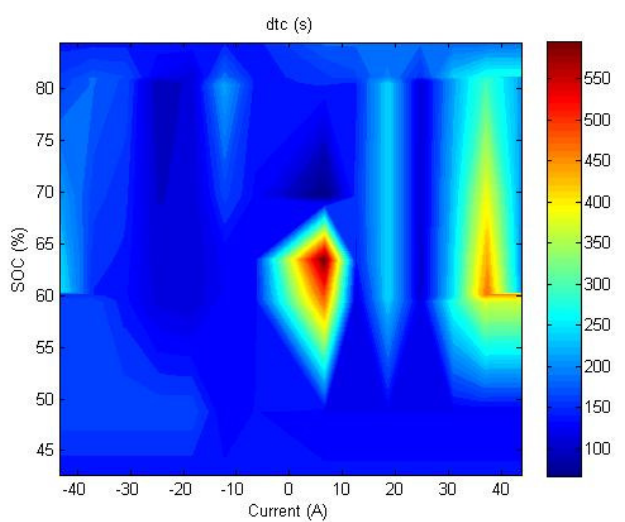

Figure 15a: Diffusion Time Constant (dtc) of the VL6P battery at the beginning of the ageing cycle

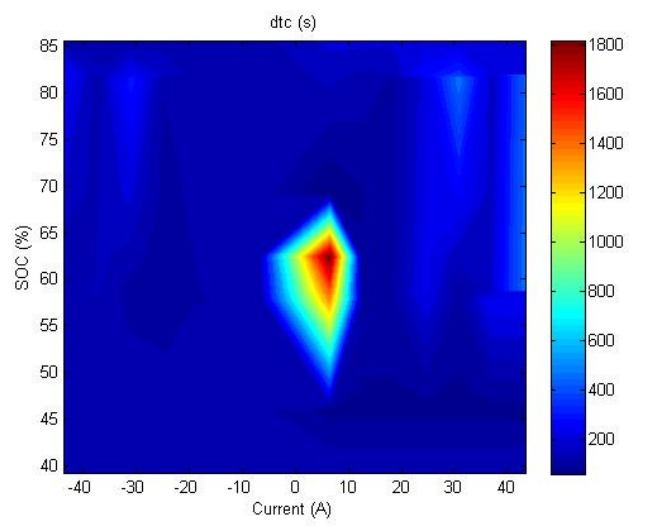

Figure 15b: Diffusion Time Constant (dtc) of the VL6P battery at the middle (27 / 04 / 2009) of the ageing cycle

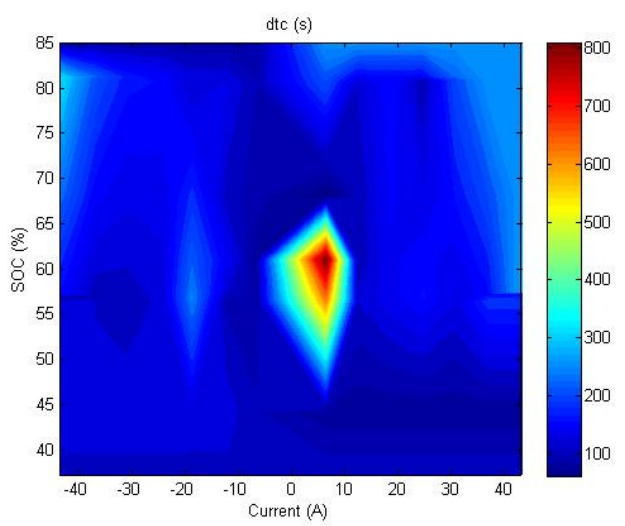

Figure 15c: Diffusion Time Constant (dtc) of the VL6P battery at the end of the ageing cycle 


\section{Conclusion}

The present paper describes the overall process in the context of the SIMSTOCK program, associating experimental and simulation tools, to follow up the ageing of a Li-Ion battery. The first step is the definition of the experimental cycle to accelerate the ageing and the characterization protocol to follow the behavioral evolution of the battery. In parallel, a numerical model battery, with an electrical approach, and its parameterization assistant are developed. The latter is able to determine the evolution of the parameters of the numerical model as a function of the ageing of the battery. From the evolution of parameters identified, thanks to the theory of experiment designs, it will be possible to determine the ageing laws of different parameters involved in the numerical model. The last step of the program will be to adapt the present model to take into account the impact of these ageing laws.

The SIMSTOCK program concentrates its research on the ageing of a battery during cycling. Nevertheless, the network partners are currently involved in the initiation of the program SIMCAL. This consists in following the same process as SIMSTOCK by studying the ageing of batteries in calendar conditions.

\section{Acknowledgments}

From the corresponding author to all the SIMSTOCK network partners who have participated: BATSCAP: O. Caumont, CEA: Y. Reynier, EIGSI: A. Mieze, EDF: L. Jamy, IFP: F. Badin, INRETS LTE: S. Pélissier, INRETS LTN: J. Kauv, IMS: J.M. Vinassa, LEC: C. Forgez, LRCS: C. Delacourt, MTA Cluster: C. Adès, PSA: D. Porcellato, RENAULT: P. Gyan, SAFT: S. Bourlot, VALEO: D. Benchétrite, ADEME: S. Biscaglia.

The authors also wish to thank the French ADEME for their active support in this research.

\section{References}

[1] J. Vetter, P. Novák, M.R. Wagner, C. Veit, K.C. Möller, J.O. Besenhard, M. Winter, M. WohlfahrtMehrens, C. Vogler and A. Hammouche, Ageing mechanisms in lithium - ion batteries, J. Power Sources, 147, 2005, pp: 269-289.
[2] R. Spotnitz, Simulation of capacity fade in lithium ion batteries, J. Power Sources, 113, 2003, pp: 72-80.

[3] P. Arora, R.E. White, M. Doyle, Capacity fade mechanisms and side reactions in lithium - ion batteries, J. Electrochemistry Society, Vol. 145, No. 10, Oct. 1998.

[4] Livrables SIMSTOCK rapport $n^{\circ} 1$.

[5] F. Badin, Modelling of On-board Energy Storage System ageing. The French SIMSTOCK research network, EVS 24, The International Battery, Hybrid and Fuel Cell Electric Vehicle Symposium \& Exhibition, Stavanger (Norway), May 2009.

[6] J. Khum, C. Forgez, P. Lagonotte, G. Friedrich, Modelling Ni-MH battery using cauer and foster structures, Journal of Power Sources, 17, 2005.

[7] J. Hafsaoui, J. Scordia, F. Sellier, and P. Aubret, Development of an electrochemical battery model and its parameters identification tool, S.A.E Society of Automotive Engineers World Congress - Yokohama (Japan), May 2010.

\section{Authors}

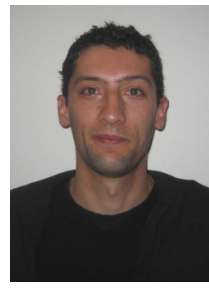

\section{Ph.D, Hafsaoui Julien}

J. Hafsaoui (Ph. D), aged 30, joined LMS Imagine in 2008 as project engineer in the Internal Combustion Engine Business Unit. J. Hafsaoui has a scientific doctorate in Materials Science at the Mines ParisTech School (E.N.S.M.P), Paris, France. $\mathrm{He}$ has a five years Engineering Degree in chemistry processes at the National Polytechnic Institute in Grenoble, France.

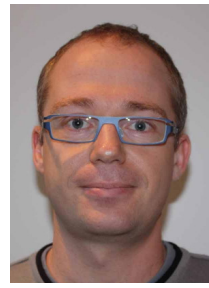

Sellier Franck

Franck Sellier joined LMS Imagine in 1999 as mechanical engineer after graduating from Ecole Centrale Marseille (former ESM2). He is the solution manager in charge of the Electric and Fluids Business Units. 